Stefanie Steinebach (2009): „Da willst Du wirklich hin? Als Frau, so ganz alleine?“ - Geschlecht im Feld. In: Elfriede Hermann, Karin Klenke und Michael Dickhardt (Hg.): Form, Macht,

Differenz. Motive und Felder ethnologischen Forschens. Göttingen: Universitätsverlag Göttingen, 371-385. Doi: 10.17875/gup2020-1298

\title{
„Da willst Du wirklich hin? Als Frau, so ganz alleine?"“ - Geschlecht im Feld
}

\author{
Stefanie Steinebach
}

„Da willst Du wirklich hin? Als Frau, so ganz alleine? Im Regenwald?“ stirnrunzelnd und kopfschüttelnd betrachteten mich meine Mutter, Schwester, Freundinnnen und Freunde, als ich sie über meine geplante Feldforschung im Regenwald von Sumatra informierte. Verwöhnt durch ein akademisches Umfeld, in dem die Rezeption (de)konstruktivistischer Geschlechtertheorien mittlerweile zur Etablierung von Genderstudies oder Geschlechterforschung als eigenen Studiengängen geführt hat, schienen mir auf meinem Geschlecht basierende Zweifel an meinem Vorhaben fast als Unverschämtheit.

Die Bedeutung des Geschlechts während der Feldforschung, vor allem hinsichtlich der Möglichkeiten und Restriktionen für die teilnehmende Beobachtung, wurde bereits vielfach in der ethnologischen Literatur diskutiert. Brigitta Hauser-Schäublin (2002) beispielsweise betrachtet dieses Thema in ihrem Artikel „Gender: Verkörperte Feldforschung" vor dem Hintergrund ihrer eigenen 30jährigen Forschungserfahrungen in unterschiedlichen Kontexten. Mit diesem und anderen Artikeln zum Thema Geschlecht im Feld hatte ich mich vor meinem eigenen Feldaufenthalt in Sumatra intensiv beschäftigt und fühlte mich bestens vorbereitet. Die Komplexität meiner Genderidentität und ihre Bedeutung für den Verlauf der Forschung sowie die daraus resultierenden Ergebnisse wurden mir jedoch erst während meines Feldaufenthaltes deutlich. In diesem Beitrag werde ich die Bedeutung von Gender und den Prozessen des Aushandelns der eigenen Genderidentität als Ethnologin im Feld vor dem Hin- 
tergrund meiner Forschung über die Konstruktion ethnischer Identität bei den Orang Rimba in Sumatra reflektieren.

\section{Das Feld}

Von 2003 bis 2005 untersuchte ich auf Sumatra, Indonesien, die Bedeutung des Regenwaldes in der Konstruktion der ethnischen Identität der Orang Rimba. Die insgesamt etwa 2000 Orang Rimba leben als semi-nomadische Regenwaldbewohner in der Bukit Duabelas Region der Provinz Jambi in Zentralsumatra. Sie unterteilen die Welt in ein Innerhalb und ein Außerhalb des Regenwaldes, wobei der Regenwald, rimba, von der Welt außerhalb des Regenwaldes, der dunia luar (Außenwelt) oder dunia terang (helle, klare Welt) umgeben ist. Aus ihrer Lebensweise im tropischen Regenwald ergibt sich für die Orang Rimba ihre Eigenbezeichnung „Orang Rimba“, Regenwaldmenschen (orang $=$ Mensch, rimba $=$ Regenwald), als logische Konsequenz. Der Regenwald als Lebenswelt stellt sich als Gefüge verschiedener sich durchdringender Ebenen dar, die das adat ${ }^{1}$ und damit die soziale und kulturelle Praxis der Orang Rimba bestimmen. Der Regenwald bildet als Rahmen für Bedeutungen und Handlungen eine komplexe Welt, in der die Orang Rimba zu einer Einheit mit dem Regenwald werden und in der sowohl sämtliche Formen der Interaktion als auch die Existenz der Orang Rimba selbst vom Regenwald determiniert werden. In diesem Zusammenhang geht die Bedeutung des Regenwaldes für die Orang Rimba weit über seine materielle Bedeutung als Subsistenzgrundlage hinaus. Das im Regenwald verortete adat der Orang Rimba als Imperativ sozialer Handlungen wird dabei zu einem Garant der moralischen Ordnung und der Regenwald somit zu dem existentiellen Referenzpunkt der eigenen ethnischen Identität.

Als Lebenswelt wird der Regenwald zu einem Ort, dessen Struktur und Organisation sich deutlich von der dunia terang, dem Nicht-Regenwald unterscheidet. Entsprechend der Unterteilung der Welt in ein Innerhalb und ein Außerhalb des Regenwaldes findet auch eine Kategorisierung der Bewohner beider Welten durch die Orang Rimba statt. Die außerhalb des Regenwaldes lebenden Menschen werden kollektiv als orang luar (Außenmenschen), orang terang (helle, klare Menschen) oder auch orang dusun (Dorfmenschen) bezeichnet. Die Zugehörigkeit zu einer ethnischen Gruppe wird von den Orang Rimba nicht durch Abstammung oder verwandtschaftliche Beziehungen, sondern durch den Lebensort der jeweiligen Person bestimmt.

Das von den Orang Rimba bewohnte Regenwaldgebiet ist umgeben von Ölpalmund Kautschukplantagen sowie Dörfern und Siedlungen der autochthonen Bevölkerung sowie der vorwiegend javanischstämmigen (Arbeits-)Migranten, die alle jeweils spezifische Nutzungsansprüche an den Wald formulieren. Im Jahr 2003 wurde das Waldgebiet auf Druck von Naturschutzorganisationen zum Nationalpark mit einer Größe von ca. 65.000 ha erklärt, um weitere Rodungen des Tieflandregenwaldes zu 
verhindern. Vor diesem Hintergrund untersuchte ich die Bedeutung des Regenwaldes in der Konstitution der Identität der Orang Rimba und anderer Gruppenidentitäten sowie die daraus resultierenden politischen Aktions- und Artikulationsmöglichkeiten der Orang Rimba, die aufgrund ihrer Lebensweise politisch und gesellschaftlich marginalisiert sind und deren Lebensraum trotz seiner Ausweisung als Schutzgebiet ${ }^{2}$ permanent durch Abholzung bedroht ist.

Die Betrachtung von ethnischer Identität als einem dialogischen Prozess von Eigenund Fremdkategorisierungen zwischen verschiedenen Gruppen erforderte meine Interaktion nicht nur mit den Orang Rimba, sondern auch mit den anderen Akteursgruppen und Einzelpersonen außerhalb des Nationalparks. So haben im Verlauf meiner Feldforschung über knapp 15 Monate hinweg immer wieder Begegnungen mit neuen Menschen in direktem und indirektem Zusammenhang mit meinem Forschungsthema stattgefunden - mit Freunden, bei Behörden, beim Einkaufen und unterwegs - die jedes Mal eine weitere Facette in der Bedeutung des Regenwaldes für die Konstruktion ethnischer Identität aufzeigten.

\section{Geschlecht im Feld}

Während der Begegnungen mit all diesen unterschiedlichen Menschen war es jedoch nie nur ich, die Fragen stellte und Antworten suchte. Immer war auch ich gefordert, Auskunft über mein Tun, meinen kulturellen, beruflichen und privaten Hintergrund etc. zu geben, um für meine Gesprächspartner einschätzbar und kategorisierbar zu werden. Dabei spielte mein Geschlecht als ein wichtiges Moment meiner Identität in Verbindung mit anderen Kriterien der sozialen Distinktion wie Herkunft/Nationalität, Hautfarbe, Religion, Beruf etc. und die damit verbundenen Erwartungen an mich eine entscheidende Rolle. Denn so wie die ethnische Identität immer ein Prozess der Eigen- und Fremdkategorisierung ist (Jenkins 1997), ist auch die personale Identität der Ethnologin ein Akt gegenseitiger Zuschreibungen zwischen Forscherin und Beforschten. Erst durch den mir zugeschriebenen Status (oder meine Identität) als Deutsche, (unverheiratete) Frau, Wissenschaftlerin und Ethnologin wurde ich zu einer Person, der bestimmte Informationen zugänglich gemacht oder verwehrt wurden. Dabei unterschieden sich die mir zugeschriebenen Rollen und die damit verbundenen Erwartungen an mein Verhalten je nach Akteuren mitunter deutlich voneinander, eine Bezugnahme auf mein Geschlecht fand jedoch direkt oder indirekt immer statt: „Feldforschung bedeutet immer Verkörperung und Verkörperung bedeutet Rückbezug auf Geschlecht und Alter, so wie diese von den Handelnden selbst dargestellt und von den anderen wahrgenommen werden", wie Hauser-Schäublin (2002:75) schreibt. Eine Reflektion der Bedeutung des Geschlechts bzw. der sozialen Kategorie „Frau im Feld“ erfordert also die Auseinandersetzung mit Gleichheit und Verschiedenheit, mit Differenz zwischen und innerhalb von Geschlechtergruppen. Dazu werde ich Geschlecht im 
Feld aus drei Perspektiven betrachten: Feldforschung als Frau, Feldforschung mit Frauen und Feldforschung von Frauen im Sinne der ethnographischen Repräsentation.

\section{Feldforschung als Frau}

Um meine Feldforschung im Bukit Duabelas Gebiet durchführen zu dürfen, musste ich verschiedene Behörden besuchen, um mich vorzustellen oder Genehmigungen zu beantragen. Die dort geführten Gespräche liefen fast immer nach demselben Muster $\mathrm{ab}$. In Indonesien ist es üblich, bevor man zum eigentlichen Anliegen des Besuches kommt, einen mehr oder weniger ausgedehnten Smalltalk zu führen, der in der Regel auch bestimmte ,persönliche Fragen wie z. B. Alter, Familienstand, Herkunft, aber auch gemeinsame Bekannte etc. umfasst. Durch meine Herkunft aus Deutschland waren Fragen nach Fußball ebenfalls Standard, gefolgt von dem Thema „Freier Liebe“ und meinem Vorhaben, mit den Orang Rimba zu leben. Exemplarisch gebe ich einen Ausschnitt aus meinem Tagebucheintrag wieder, den ich nach meinem Besuch der Polizeistation einer Kleinstadt in Sumatra verfasst habe.

[Tagebucheintrag, 15. Februar 2004 / Polizeistation]

Heute besuchte ich die Polizeistation in Bangko, um dort meinen Aufenthalt in der Region zu melden. Wieder musste ich mich über Fußball unterhalten - was indonesische Männer mit Deutschland assoziieren, scheint in erster Linie Fußball zu sein. Ich habe von Fußball keine Ahnung, Fußball interessiert mich nicht! Sollte er aber... Meine Gesprächspartner korrigierten mich geduldig, wenn ich wiederholt Spieler und Vereine in der falschen Kombination nannte. Dieses Unwissen ließ sich nur dadurch entschuldigen, dass ich glaubhaft versichern konnte, dass Fußball in Deutschland Männersache ist, und eine „richtige“ Frau von Fußball keine Ahnung haben sollte, möchte sie sich nicht als ,kampungan' oder ,tomboi“ zu erkennen geben. ${ }^{3}$ Ich bin schließlich eine richtige Frau! Während ich dies vehement vertrete, bete ich inständig, dass keine meiner Freundinnen und Kolleginnen in Deutschland mich hören kann $[\ldots]$.

In diesem Gesprächsausschnitt wird deutlich, dass auf der Polizeistation meine Nationalität das zentrale Kriterium ist, anhand dessen eine Identifikation und soziale Kategorisierung stattfindet. Die an meine Herkunft geknüpfte Erwartung ist eine Kompetenz in Sachen Fußball. Die Enttäuschung dieser Erwartung erkläre ich mit der Bezugnahme auf mein Geschlecht und die damit angeblich in meiner Heimat verbundenen Rollenvorstellungen. Die Betonung meiner Genderidentität als „richtige“ Frau verwendete ich in dieser Situation strategisch, um die Geschlechterdifferenz zwischen mir und meinen Gesprächspartnern zu manifestieren und damit meine Moral und meinen Anstand im Umgang mit Männern zu betonen. In Deutschland hingegen empfinde ich es durchaus 
als positiv, wenn Frauen sich die Männerdomäne Fußball erobern und so Geschlechterrollen und Hierarchien in Frage stellen. Mein Rekurs auf klassische Rollenverteilungen auch im Bezug auf Sport entspricht so nicht meinem Ideal von emanzipativer Geschlechterdekonstruktion wie ich sie im deutschen Alltag propagiere. Gleichzeitig wird deutlich, dass sich für meine Gesprächspartner meine nationale Identität vor die geschlechtliche schiebt, denn auch in Indonesien gilt das Interesse für Fußball als spezifisch männliche Domäne und eignet sich keinesfalls dafür, sich als „echte“ Frau zu beweisen.

Auch im zivilen Rahmen und mit Frauen verliefen die Gespräche ähnlich wie das auf der Polizeistation. Allerdings wurde hier mein Frausein als Gemeinsamkeit stiftendes Moment in den Vordergrund gestellt. Schnell zeigten sich jedoch Unterschiede beispielsweise in den Vorstellungen und Erwartungen darüber, wie eine ,moderne Frau zu sein hat - ein Thema, das gerne von meiner ökonomisch sehr gut situierten Gastfamilie in der Kleinstadt Bangko diskutiert wurde. Ich illustriere ein solches Gespräch durch die Wiedergabe einer Passage aus meinem Tagebuch:

[Tagebucheintrag, 15. Februar 2004 / Kel. Armen]

Nachdem ich meine auf der Polizeistation frisch erworbenen Kenntnisse der deutschen Fußball Bundesliga und ihrer Protagonisten souverän und ohne größere Fehler mit dem Hausherren diskutiert hatte, wurde ich zum Gespräch mit Frau und Töchtern des Hauses entlassen.

Aber auch in der folgenden Unterhaltung stellt sich schnell heraus, dass ich zwar von Fußball recht wenig Ahnung habe, davon, was es heißt eine ,richtige Frau“ zu sein aber auch. Das ließ sich anhand folgender Problemfelder erkennen: Ich bin nicht verheiratet - und das in meinem Alter (Jahrgang 1972). Das ist ungewöhnlich und veranlasst die Frauen des Hauses zu verschiedenen Mutmaßungen: Ein Grund dafür, dass ich noch nicht verheiratet bin, könnte meine Kleidung sein - viel zu männlich, oder doch eher ,kampungan ${ }^{4}$. Ich sollte mich weiblicher und auch schicker kleiden, leisten könnte ich es mir als Wissenschaftlerin schließlich!

Zusätzlich stellte sich recht bald heraus, dass es auch mit meinen Kenntnissen über Make-up und die richtige Verwendung der einzelnen Schminkutensilien über gängige Praktiken hinaus nicht allzu gut bestellt ist. Glücklicherweise habe ich eine Tante, die lange als Model Tätig war und in der Modebranche beschäftigt ist. Schnell holte ich einige Fotos von meiner attraktiven, blonden, perfekt geschminkten und gekleideten Tante hervor. Bewundernde „ahs“, und „ohs" kommentieren die Bilder, die erfolgreich von meiner unzulänglichen Rollenperformanz als gebildete Mittelschichtsfrau ablenkten und ein bisschen von dem Glanz meiner Tante auch auf mich übergehen ließen. So konnte anhand verwandtschaftlicher Bande belegt werden, dass auch in mir irgendwo wenigstens die Anlagen zur richtigen Frau schlummern $[\ldots]$. 
Diese Begebenheiten fanden in einem kleinstädtischen Umfeld von Beamten und Mittelschichtsbürgern statt. Die lokalen Entwürfe von Männlichkeit und Weiblichkeit waren zudem geprägt von global zirkulierenden Geschlechterbildern in den Medien (vgl. Hauser-Schäublin und Braukämper 2002:11), die oft ein Bild westlicher Frauen als unmoralisch zeichneten (vgl. Schlehe 2002), das als negative Folie für den offiziellen (staatlichen) Diskurs über „die indonesische“ Frau als moralisch integere, monogame Gattin dient.

Während ich als Frau also an divergierenden lokalen Konzepten von Weiblichkeit in Verbindung mit bestimmten Ideen über deutsche Wissenschaftlerinnen gemessen wurde, versuchte ich gleichzeitig, diesen mit einem je spezifischen Auftreten zu entsprechen. Wie unterschiedlich diese Gender-Konzepte und die von mir geforderten jeweiligen Verkörperungen waren, möchte ich verdeutlichen, indem ich einen Einblick in meine Interaktion mit Orang-Rimba-Frauen gebe. Während ich in der Kleinstadt und bei der Dorfbevölkerung am Rand des Regenwaldes darauf insistieren musste, aktiv am Kochen, Gemüseschneiden etc. teilnehmen zu dürfen, musste ich bei den Orang-Rimba-Frauen meine „Tauglichkeit“ als Frau immer wieder durch harte Arbeit unter Beweis stellen:

Die Orang Rimba, insbesondere die Frauen, luden mich von sich aus ein, mich an der Feldarbeit und dem Jagen und Sammeln zu beteiligen. Zur Zeit der Reisernte beispielsweise stellte ich mich beim Reisstampfen recht ungeschickt an, und nach kurzer Zeit hatte ich von der ungewohnten und schweißtreibenden Arbeit offene Blasen an den Händen. Trotzdem wurde ich angehalten, diese Tätigkeit weiter auszuüben. Erst als das Blut von meinen Händen tropfte, hatten die Frauen und Mädchen ein Einsehen, und mir wurde gestattet, mit dieser Tätigkeit aufzuhören. In regelmäßigen Abständen musste ich meine Hände mit denen der Orang Rimba vergleichen lassen, um zu sehen, ob sich mittlerweile Hornhaut und Schwielen als Beweis meiner körperlichen Leistungsfähigkeit gebildet hätten.

Gerade diese Schwielen als Beweis meiner Annäherung an das Ideal einer tüchtigen, auch schwere körperliche Tätigkeiten ausübenden Orang-Rimba-Frau waren es, die mich unter anderem daran hinderten, die Ansprüche an das Ideal einer gepflegten „modernen“ Frau in meinem kleinstädtischen indonesischen Umfeld zu erfüllen. Diese konträren Diskurse über die Rollen von Frauen konstruierten auch meine Genderidentität immer wieder neu und konfrontierten mich mit unterschiedlichen Erwartungen, denen ich jedoch immer nur in bestimmtem Umfang entsprechen konnte.

\section{Ungleiche Frauen}

Deutlich wird an diesen Beispielen die Bedeutung der Differenz innerhalb der Geschlechtergruppe „Frau“s: zum einen die Differenz zwischen mir als deutscher Ethnologin und den Frauen im Feld, zum anderen die lokalen Differenzen hinsichtlich der 
sozialen Kategorie „Frau“. Diese variieren in Abhängigkeit der ethnischen und religiösen Zugehörigkeit, der Klassen- oder Schichtzugehörigkeit, dem Alter, dem Zugang zu Bildung und Information und vielen weiteren soziokulturellen Faktoren.

Die Differenz zwischen meiner ,Weiblichkeit ${ }^{\prime}$ und der lokaler Frauen zeigt sich besonders deutlich an den Möglichkeiten meiner Interaktion mit dem Geschlecht "Mann": Sowohl bei den Orang Rimba als auch in den unterschiedlichen Zusammenhängen außerhalb des Waldes wurde mir oft gestattet, an männlich assoziierten oder Männern vorbehaltenen Aktivitäten wie beispielsweise bestimmten Formen der Jagd oder am Zigarettenrauchen teilzunehmen, manchmal wurde ich gar dazu eingeladen. Hier wurde mein Geschlecht zugunsten anderer Differenzierungskriterien in den Hintergrund gestellt und mir so eine Identität als Ethnologin zugewiesen, die kontextuell am ehesten den Erwartungen und Vorstellungen meiner (männlichen) Gegenüber entsprach, die mich für die jeweiligen Frauen jedoch oft schwer einschätzbar werden ließ oder diese mir gegenüber misstrauisch machten.

Gleichzeitig ist auch der relationale Aspekt verschiedener Genderidentitäten untereinander zu beachten. ,Männlich` und ,weiblich`werden immer auch in Beziehung zueinander bzw. in Abgrenzung voneinander konstituiert, so dass die Beschäftigung mit einem Geschlecht immer auch die (indirekte) Auseinandersetzung mit dem anderen Geschlecht - oder unter Umständen alternativen Geschlechterkonzepten - bedeutet.

Im Hinblick auf eine geschlechtliche Identität im Feld wird deutlich, dass dieses „Spiel mit Identitäten“" (Hauser-Schäublin 2002:86) und auch deren Instrumentalisierung zur Verringerung der Unterschiede zwischen der eigenen Forscherinnenpersönlichkeit und ,den Anderen' durch die Geschlechterdiskurse in der untersuchten Gesellschaft bestimmt wird. So erscheint die (Gender-)Identität der Ethnologin im Feld aus einer Kombination der ,eigenen' kulturellen Spezifika der Ethnologin zu bestehen, deren jeweilige Strukturierung und Betonung jedoch durch ,die Anderen' im Bedeutungsrahmen ihres kulturellen Wertesystems erfolgt und die so letztendlich auch den Handlungs- und Aktionsradius der Ethnologin im Feld bestimmen.

(De)konstruktivistische Gendertheorien ermöglichen zwar eine differenzierte Analyse dieser Diskurse und Prozesse im Feld, sie dürfen jedoch nicht darüber hinwegtäuschen, dass die Konstruktion von Genderidentitäten (im Feld) kein individueller Akt der autonomen Ethnologin ist, sondern dass diese von lokalen Diskursen über Geschlecht in Verbindung mit Konzepten des „Anderen“ oder „Fremden“ maßgeblich beeinflusst werden.

\section{Feldforschung mit Frauen}

Dienten die vorangegangenen Beispiele der Interaktion zwischen mir und unterschiedlichen Frauen im Feld der Reflexion meiner geschlechtlichen Positionierung als Prozess zwischen Zuweisung und Aneignung, möchte ich hier ausführlicher auf die konkrete 
Forschungssituation mit Orang-Rimba-Frauen eingehen. Zuvor gebe ich einen knappen Einblick in das Geschlechterverhältnis der Orang Rimba:

Die Orang Rimba unterscheiden zwei Geschlechter, männlich (genton) und weiblich (betina). Dabei kommt dem weiblichen Geschlecht in der egalitären Orang Rimba Gesellschaft ein hoher Stellenwert zu. Die Orang Rimba leben in verwandtschaftlich organisierten Gruppen von ca. 15 bis 35 Personen, deren Zusammensetzung durch die matrilineare Abstammungs- sowie die matri- und uxorilokale Wohnsitzregelung der Orang Rimba bestimmt wird. So bleiben die Frauen einer Verwandtschaftsgruppe ihr Leben lang zusammen, während die Söhne in andere Gruppen heiraten. Ab etwa dem siebten Lebensjahr sind die Kontakte zwischen Jungen und Mädchen streng geregelt, Umgang miteinander ist, wenn überhaupt, nur in Anwesenheit Dritter erlaubt. Ab diesem Alter gelten die Jungen bereits als „Junggesellen“ und schlafen nicht mehr im selben Haus mit ihren Eltern und Schwestern.

Haben ein junger Mann und eine junge Frau Interesse aneinander, werden die möglichen verwandtschaftlichen Beziehungen zwischen beiden überprüft. Lassen die verwandtschaftlichen Beziehungen junger Heiratswilliger eine Eheschließung zu, muss der potentielle Bräutigam einen Brautdienst bei seinen zukünftigen Schwiegereltern absolvieren. Während eines Zeitraumes von einem bis zu mehreren Jahren muss der junge Mann seinen Fleiß und seine Fähigkeiten beweisen, eine Familie zu ernähren, indem er seine Arbeitskraft der Familie seiner zukünftigen Ehefrau ohne Gegenleistung zur Verfügung stellt. Verläuft die Zeit des Brautdienstes zufriedenstellend für alle Beteiligten und ist die Heirat gewünscht ${ }^{6}$, wird zwischen den Familien der Braut und des Bräutigams ein Brautpreis ausgehandelt, der in der Regel für die jüngste Tochter einer Familie am höchsten ausfällt. ${ }^{7}$ Als optimale Verbindung wird die als juwor dengan palau ${ }^{8}$ bezeichnete matrilaterale Kreuzcousinenheirat betrachtet.

Nach der Heirat ist es den Ehemännern nicht mehr gestattet, länger als drei Nächte von ihrer Frau getrennt zu sein. Die Nichteinhaltung der Eheverpflichtungen kann entweder nach dem adat bestraft oder auch als Scheidungsgrund gewertet werden. Die Scheidung (bototoruwtowon - Durchtrennen des Rattan) kann sowohl von Ehemännern als auch -frauen gewünscht werden. Kommt es zur Scheidung, muss von Männern eine Strafe von 20 kain (Batikstoffe, die bei den Orang Rimba als Währung verwendet werden) von Frauen hingegen eine Strafe 40-60 kain gezahlt werden. Die höhere Strafe für Frauen ergibt sich dadurch, dass Männer allgemein mit Verlust aus einer Scheidung hervorgehen. Durch die uxorilokale Wohnsitzregelung verlieren die Männer ihren Wohnort, die Kinder bleiben bei der Mutter, immobiler Besitz wie etwa Honigbäume wird matrilinear vererbt, so dass nach einer Scheidung der Mann auch hier die Nutzungsrechte verliert.

Bei der Verhandlung politischer Entscheidungen, die in der Regel durch Konsensfindung getroffen werden, sind Frauen und Männer in gleichem Maße stimmberechtigt und beteiligt. ${ }^{9}$ Ein Beispiel dafür sind folgende Verhandlungen über zwischen- 
geschlechtliche Interaktionen vor der Ehe, die ich in einem kurzen Auszug aus meinen Notizen wiedergebe:

[Tagebucheintrag 31. Januar 2005, Kedundung Jehat]

[...] dort [im Wald] angekommen, erfahren wir, dass es abends eine Versammlung geben soll wo 2 Fälle von vorehelicher Kontaktaufnahme ${ }^{10}$ zwischen zwei jungen Menschen vorgefallen sind, die nun in der Orang Rimba Gemeinschaft verhandelt werden, da sie gegen das adat verstoßen haben.

Die männliche Verwandtschaft des Mädchens (wari betina) muss die Position des Mädchens vertreten. Andererseits sind auf der Seite des Mannes die Frauen für die Vertretung zuständig. Im aktuellen Fall hatte der Mann bereits seit 2 Jahren Brautdienst bei der Familie der Frau geleistet, erzählt nun überall herum, dass er sie schon hätte baden sehen und andere Intimitäten, die erst nach der Eheschließung gestattet sind. Das Mädchen widerspricht diesen Gerüchten, aber die sind schon zu weit herumgegangen, so dass der Fall nun verhandelt werden muss. Für die Seite des Mädchens kommt eine Eheschließung nicht in Frage, sie sagen das wäre Inzest ${ }^{11}$ und fordern 20 koping kain bersih. Das heißt, 20 Stoffe und keine Heirat. Die Seite von Ngrip ist aber nur bereit, 2 kupang zu zahlen, was im Endeffekt auf 2 Stoffe hinausläuft.

Während der Verhandlungen geht es sehr lebhaft zu, ganze Familienverbände sind anwesend, diejenigen, die gerade nicht aktiv in den Verhandlungen involviert sind, kochen. Während der Wortführung, dem Aushandeln der Strafe, sind sowohl die Frauen als auch die Männer involviert. Die Frauen äußern sich lautstark, am Rande des Geschehens fordert Besmi Ngrip zum Boxen heraus... - es geht hoch her, aber danach, sobald das Thema gewechselt wird, sind alle wieder die besten Freunde. Es ist das übliche „Theater“. [...]

Dann kommt Temenggung Mirak [ein Mann] mit Frau und Kindern, um den zweiten Fall, Betatai [ein Junggeselle] mit einem Mädchen aus der Familie Pengendums, zu verhandeln. Betatai hat der Familie 2-3 Tage nugal (Pflanzen mit dem Pflanzstock) geholfen, und dann den Ring seiner Angebeteten an sich genommen (hat sie ihn ihm gegeben oder hat er ihn einfach genommen?). Nun sollen sie schnell verheiratet werden, aber das Mädchen möchte nicht.

Insgesamt sind immer die sogenannten wari zuständig, die der Männer und Frauen, auf der Seite des Mannes ist also die weibliche Verwandtschaft, auf der Seite der Frau die männliche Verwandtschaft für die Konfliktparteien [...] zuständig.

Während Männer und Frauen im Regenwald gleichberechtigte Verhandlungspartner in soziopolitischen Belangen darstellen, ist dies außerhalb des Regenwaldes nicht immer der Fall. Der Kontakt zwischen Orang Rimba und der sesshaften Bevölkerung fand bis in die 1980er Jahre hinein nur zum Zweck ökonomischer Transaktionen und nur über einen Mittelsmann aus der sesshaften Bevölkerung, den jenang, ${ }^{12}$ statt. Durch 
die Reduktion der Waldflächen, die Besiedlung der Waldrandzonen und damit einhergehende soziopolitische und ökonomische Veränderungen und Möglichkeiten finden Kontakte zwischen den Menschen innerhalb und außerhalb des Regenwaldes heutzutage häufiger statt, sind jedoch für beide Gruppen noch immer keine Selbstverständlichkeit.

Aufgrund des adat der Orang Rimba, aber auch durch historische Ereignisse wie z. B. die Sklavenjagd in den Wäldern der Bukit Duabelas Region ist es für männliche Nicht-Orang Rimba nahezu unmöglich, generell Kontakt zu Orang Rimba-Frauen zu bekommen ${ }^{13}$ oder gar mit ihnen zu forschen (vgl. Sandbukt 1984, 1988). Diese Situation, wie sie Ethnologinnen wie beispielsweise Margret Mead im Hinblick auf ihre männlichen Kollegen vor fast 40 Jahren erlebten (vgl. Hauser-Schäublin 2002), ist im Bukit Duabelas Gebiet noch heute (2009) aktuell.

Um den Kontakt mit den eigentlich akephalen Orang Rimba für sich zu erleichtern, haben Teile der lokalen autochthonen Bevölkerung ihre eigene politische Struktur und die darin verankerten Ämter einfach auf einzelne Orang Rimba Gruppen übertragen. Dabei wurden und werden diese Ämter in Bezug auf die Struktur der sesshaften Bevölkerung nur an Männer vergeben. Auch heute noch bestimmen die Dorfvorsteher einen oder mehrere Orang-Rimba-Männer als Ansprechpartner einer Gruppe, indem sie ihnen ein Uniformhemd überreichen, durch das die jeweiligen Männer dann zu offiziellen Vertretern ihrer Gruppen werden. ${ }^{14}$

Orang-Rimba-Frauen werden keine Interaktions- und Verhandlungspartnerinnen, da in ihrer eigenen Gesellschaft Frauen den Kontakt mit Männern meiden, die nicht Orang Rimba sind, und andererseits die sesshafte Bevölkerung Frauen nicht als Inhaberinnen dieser politischen Ämter und als Verhandlungspartnerinnen vorsieht. Zusätzlich erschweren bestimmte kulturelle Praxen der Orang Rimba die Akzeptanz von Frauen in politischen Ämtern, da beispielsweise Orang-Rimba-Frauen, sobald sie verheiratet sind, ihre Brüste in der Öffentlichkeit nicht mehr bedecken (müssen), was von einem Großteil der Nicht-Orang Rimba als Ausdruck von Primitivität, sexueller Freizügigkeit und unmoralischem Denken und Handeln bewertet wird. Die Interaktion und der Prozess des Aushandelns der ethnischen Identität zwischen der sesshaften Bevölkerung und den Orang Rimba finden somit fast ausschließlich zwischen Männern der unterschiedlichen Gruppen statt. Alle Informationen, die Orang-Rimba-Frauen über die Politik außerhalb des Waldes erhalten, bekommen sie aus zweiter Hand und aus einer männlichen Perspektive.

Es sind die Männer, die sich außerhalb des Waldes ein Netzwerk von Beziehungen aufbauen und die in der Interaktion mit Menschen außerhalb der eigenen ethnischen Gruppe Erfahrungen sammeln - und die so auch aktiv die Konstruktion ethnischer Identität als Prozess zwischen Gruppen gestalten.

Die Transformation meiner Feldforschungserfahrungen mit Orang-Rimba-Frauen in die Argumentation meiner Dissertation führt schließlich zum letzten Punkt, dem Thema der Feldforschung von Frauen. 


\section{Feldforschung von Frauen}

Nach dem Feldaufenthalt erfolgen in der Regel die Auswertung des Datenmaterials und eine Verschriftlichung der Erfahrungen in Form einer Dissertation, eines Buches, Artikels oder Forschungsberichtes. Das in diesem Rahmen produzierte Wissen ist ebenso individuell wie die (Gender-)Identität der Ethnologin im Feld. Gerade deshalb wird von einer Ethnologin eine Auseinandersetzung mit der Komplexität des Themas Geschlecht erwartet, die sich als differenzierte Position diesbezüglich in ihren Schriften wieder finden sollte, möchte sie sich nicht dem Vorwurf der feministischen Abstinenz oder gar der Reproduktion des male bias aussetzen. Aus der Kombination der Fragestellung meiner Dissertation mit den Geschlechterrollen vor Ort wurde die Repräsentation von Frauen als Akteurinnen jedoch eine besondere Herausforderung.

Im Rahmen meiner Forschung zur ethnischen Identität der Orang Rimba und der Betrachtung ihrer Interaktion mit den unterschiedlichen Akteuren außerhalb des Regenwaldes verbrachte ich sehr viel Zeit mit Männern. Sie begleitete ich zu Treffen mit Dorfvorstehern, mit Forstbehörden und auch mit Nicht-Regierungsorganisationen. Die von mir dargestellten Prozesse der Konstruktion ethnischer Identität sind somit Aushandlungspraxen zwischen den Männern verschiedener (ethnischer) Gruppen. Dadurch ergibt sich letztendlich in meiner Dissertation ein male bias der repräsentierten Meinungen und Aussagen, der jedoch nicht die Gesellschaftsstruktur und Machtverhältnisse im Alltag der matrilinearen und matrilokalen Orang Rimba widerspiegelt. ${ }^{15}$

Umso deutlicher wird jedoch, dass das Feld der politischen Auseinandersetzung um den Bukit Duabelas Regenwald männlich dominiert ist. Daraus ergibt sich für mich die Frage, inwieweit durch das ausschließliche (Ver)Handeln männlicher Protagonisten in wichtigen politischen und gesellschaftlichen Belangen außerhalb des Regenwaldes das bislang mindestens gleichberechtigte Geschlechterverhältnis der Orang Rimba in Zukunft ins Wanken zu geraten droht.

Als Konsequenz für die schreibende Ethnologin bedeutet dies, die Repräsentation der Geschlechter und Geschlechterverhältnisse immer auch unter dem Aspekt der Macht zu betrachten: Wessen ethnische Identität es ist, über die ich schreibe, welche Machtstrukturen werden reproduziert und inwieweit kann die Repräsentation der feldforschenden Ethnologin Genderbeziehungen und Machtverhältnisse zwischen den Geschlechtern der beforschten Gesellschaft beeinflussen? Eine Auseinandersetzung mit Geschlecht und Geschlechterverhältnissen im Feld umfasst die Analyse von Beziehungen und Prozessen, die zeitlich und räumlich über die konkrete Forschungssituation hinausgehen. Dabei bleibt die Ethnologin auch nach der Feldforschung immer noch Teil dieser Beziehungen mit all ihren Auswirkungen.

Die zu Beginn dieses Artikels gestellte Frage nach der Bedeutung des Geschlechts im Feld hinsichtlich habe ich unter den drei Aspekten Feldforschung als Frau, Feldforschung mit Frauen und Feldforschung von Frauen betrachtet. Immer spielt das 
Geschlecht der Ethnologin eine zentrale Rolle. Immer wieder werden unterschiedliche Erwartungen mit der Rolle der weiblichen Ethnologin verbunden, so dass die Konstruktion der Genderidentität der Ethnologin im Feld ein andauernder Prozess ist, der maßgeblich bestimmt wird von lokalen Diskursen über Geschlecht und Geschlechterrollen, die nicht unbedingt mit dem Selbstbild der Ethnologin übereinstimmen. Die aus der Feldforschung resultierenden Daten sind ebenso als komplexe Ergebnisse von Geschlechterbeziehungen, Machtstrukturen und Diskursen zu betrachten. Die letztendlich stattfindende Repräsentation und Veröffentlichung der Forschungsergebnisse manifestiert und verkörpert so auch die Genderidentität der Ethnologin, in meinem Fall die Identität einer „Frau“ alleine im Regenwald.

Als Resümee dieser Selbstreflexion in Bezug auf Geschlecht im Feld zeigt sich für mich, dass eine Auseinandersetzung mit Geschlecht im Feld, wie sie Hauser-Schäublin unter anderem retrospektiv im Jahr 2002 veröffentlicht hat, weiterhin notwendig und hoch aktuell ist - und auch in Zukunft sein wird!

\section{Anmerkungen}

1 Als adat wird in Indonesien die Gesamtheit von Regeln und Normen bezeichnet, die das gesellschaftliche Zusammenleben der Gemeinschaft bestimmen. Das jeweilige adat einzelner ethnischer Gruppen basiert auf gemeinsamen Werten, Idealen und Wissen der Mitglieder dieser Gruppen, und steht so in einem engen Zusammenhang mit ihren kosmologischen und religiösen Uberzeugungen. „In a more profound sense than its usual rendering as ,customary law', adat must also be understood to incorporate the moral ideal of social consonance and the behavioural imperative of propriety. There is an underlying religious-social vision of the necessary correspondence of cosmic and human relationship towards which it is directed" (Warren 1993:3). Viele Elemente des adat-Systems sind in das alltägliche Handeln integriert und regeln beispielsweise Besitzverhältnisse, Rituale, Kleidungsvorschriften, Heiratsbeziehungen, Austauschverhältnisse, Vererbung, Titel sowie Macht- und Entscheidungsstrukturen. Das adat wird meist mündlich überliefert, wodurch es flexibel an Entwicklungen und Veränderungen angepasst werden kann. Dabei findet jedoch keine Änderung seiner Eigenschaft (sifat), d. h. seines verbindlichen und normativen Charakters, an sich statt. Das adat-Recht steht häufig als Parallelstruktur neben dem kodifizierten staatlichen Recht und religiösen Rechtsnormen. Das adat wird als Komplex gruppenspezifischer kultureller Äußerungen und Handlungen zu einem wichtigen Moment der ethnischen Identitätsformulierung und zu einem Differenzierungs- und Abgrenzungskriterium gegenüber anderen Gruppen.

2 Auch die Ausweisung des Nationalparks hat negative Implikationen für die Orang Rimba, da ihre bisherige Nutzung des Waldes als Lebensraum durch die Nationalparkgesetze extrem eingeschränkt wird.

3 Als „kampungan" (kampung = Dorf) werden umgangssprachlich als ,ungebildet" empfundene Frauen bezeichnet, die in der Regel aus ländlichen Regionen stammen und nicht dem Ideal einer modernen, gebildeten Mittelschichtsfrau entsprechen. Die lässt sich vor allem auch an der äußeren Erscheinung erkennen, wie mir erklärt wurde. 
Als „tomboi“ gilt ein Mädchen oder eine Frau, die in ihrem Rollenverhalten mitunter eher männlich orientiert ist, was sich z. B. in Freizeitaktivitäten (etwa Bergsteigen oder Waldaufenthalte) oder ähnlichem zeigt.

4 Zum Zeitpunkt des Gesprächs trug ich einen schlichten Rock und eine Bluse, wenn ich aber eine von einem Aufenthalt im Wald zurück in die Kleinstadt kam, war ich meist mit Hose und T-Shirt bekleidet, deren Sauberkeit durch den Wald und die Reise erheblich gelitten hatte.

5 Die Unzulänglichkeit des Konzeptes „Frau“ als universaler Sammelkategorie, die die Heterogenität und ungleiche Machtverhältnisse zwischen Frauen verschleiert, wurde bereits von „women of colour" und „Dritte-Welt-Feministinnen“" kritisiert (u.a. Abu Lughod 1991; Mohanty 1991, Spivak 1999, Steyerl 2003)

6 Neben diesem idealtypischen Verlauf einer Eheschließung gibt es Varianten, bei denen entweder der Brautdienst zugunsten von Geschenken (Geld und Wertgegenstände) in den Hintergrund tritt, oder aber zwei Jugendliche ohne das Einverständnis ihrer Familien gemeinsam davonlaufen. Das Ersetzen des Brautdienstes durch Geschenke findet sich vor allem in Regionen des Bukit Duabelas Gebietes, in denen Lohnarbeit und Kautschukplantagenwirtschaft größere Bedeutung für die Subsistenz haben als shifting cultivation in Kombination mit Jagen und Sammeln.

7 Bei den Orang Rimba richtet sich die Höhe des Brautpreises auch nach dem Alter des zu verheiratenden Kindes. Heiratet die jüngste Tochter (bunsunye) spricht man von einer mancung tali buoyon (cutting the swing) Heirat, für die der höchste Brautpreis zu bezahlen ist, der bis zu 60 koping kain betragen kann. Begründet wird dieser hohe Brautpreis von den Orang Rimba Eltern mit der ganz besonders großen Zuneigung, die sie für ihre jüngste Tochter empfinden, mit dem großen Schmerz, den ihnen die Trennung von ihr verursacht, und der besonderen Fürsorge, die sie in ihre Erziehung investiert haben.

8 Juwor und palau sind die Namen zweier von den Orang Rimba als äußerst delikat betrachteter Fischarten.

9 Die Gender-Konzepte der Orang Rimba sehen für den Alltag bestimmte Arbeitsteilungen, bestimmte Nahrungstabus und auch voneinander abweichendes Vokabular vor.

$10 \mathrm{Ab}$ einem Alter von sieben Jahren gelten Jungen bei der Orang Rimba bereits als Junggesellen. Sie schlafen ab diesem Zeitpunkt gemeinsam in einem Junggesellenhaus, der Kontakt zu Mädchen und unverheirateten Frauen ist streng reglementiert und darf nur im Beisein anderer (Erwachsener) stattfinden.

11 Als Inzest oder sumbang werden folgende Beziehungen angesehen:

Ego mit eigenen Kindern (anak),

Ego mit Neffen oder Nichten (keponakan),

Ego mit (Stief-)Geschwistern (saudara tiri),

Ego mit Zieh-od. Adoptivkindern (anak pengambilan, anak angkat).

Verbindungen, die als nicht optimal oder sogar als Tabu angesehen werden, werden als pancit dengan membiang bezeichnet. Pancit und membiang sind zwei Arten von Fisch, die von den Orang Rimba als ungenießbar klassifiziert werden und deren Verzehr Mädchen und Frauen verboten ist.

12 Bis in die 1970er Jahre fanden ökonomische Tauschbeziehungen zwischen den Orang Rimba und der sesshaften Bevölkerung entweder über den Mittelsmann oder in Form des ,Stillen Tausches' - silent barter statt.

13 Die Namen unverheirateter Mächen werden nicht in der Öffentlichkeit und schon gar nicht vor Fremden erwähnt, um sie vor dem Einfluss schwarzer Magie (pellet) zu schützen. Das Gleiche gilt für das Fotografieren von Mädchen und Frauen, das von den Orang Rimba in 
der Regel nicht gestattet wird. Wurden Fotos von Mädchen oder Frauen aufgenommen, so dürfen diese nur unter Absprache und in sehr seltenen Ausnahmen Dritten oder gar einer Öffentlichkeit zugänglich gemacht werden.

14 Die Vergabepraxis der Dorfvorsteher führt immer wieder zu Unmut unter den Orang Rimba, da so hierarchische Verhältnisse entstehen.

15 Die meiner Fragestellung und der lokalen Praxis geschuldete Unsichtbarkeit von OrangRimba-Frauen in meiner Dissertation führte immer wieder zu kontroversen Diskussionen im Doktorandenkolloquium - auch mit Brigitta Hauser-Schäublin. Wir einigten uns schließlich darauf, dass ich dieses Thema auch in einem separaten Artikel reflektieren könnte. Dieser Beitrag ist somit auch eine Referenz an unsere lebhaften und produktiven Diskussionen, die mich zwangen, meine diesbezüglichen Überlegungen zu schärfen.

\section{Literatur}

Abu-Lughod, Lila (1991) Writing against Culture. In: Richard G. Fox (Hg.): Recapturing Anthropology. Working in the Present, S. 137-162. Santa Fe: School of American Research Press.

Hauser-Schäublin, Brigitta (2002) Gender: Verkörperte Feldforschung. In: Hans Fischer (Hg.): Feldforschungen: Erfahrungsberichte zur Einführung (2. Auflage, Neufassung), S. 73-100. Berlin: Reimer.

Hauser-Schäublin, Brigitta (2003) Teilnehmende Beobachtung. In: Bettina Beer (Hg.), Methoden und Techniken der Feldforschung; S. 33-54. Berlin: Reimer.

Hauser-Schäublin und Ulrich Braukämper (2002) Zu einer Ethnologie der weltweiten Verflechtungen. In: Hauser-Schäublin und Ulrich Braukämper (Hg.): Ethnologie der Globalisierung. Perspektiven kultureller Verflechtungen. S. 9-14. Berlin: Reimer.

Hauser-Schäublin und Birgit Röttger-Rössler (Hg.) (1998) Differenz und Geschlecht. Berlin: Reimer.

Jenkins, Richard (1997) Rethinking Ethnicity: Arguments and Explorations. London: Sage.

Mohanty, Chandra T. (1991) Under Western Eyes: Feminist Scholarship and Colonial Discourses. In: Anne McClintock, Aamir Mufti und Ella Shohat (Hg.): Dangerous Liaisons. Gender, Nation and Postcolonial Perspectives, S. 255-274. Minneapolis: University of Minnesota Press.

Sandbukt, Øyvind (1984) Kubu Conceptions of Reality. Asian Folklore Studies 43:8598.

Sandbukt, Øyvind (1988) Tributary Tradition and Relations of Affinity and Gender among the Sumatran Kubu. In: Tim Ingold, David Riches und James Woodburn 
(Hg.): Hunters and Gatherers (Band 1: History, Evolution and Social Change), S. 107-116. New York und Oxford: Berg.

Spivak, Gayatri Chakravorty (1999) A Critique of Postcolonial Reason: Toward a History of the Vanishing Present. Cambridge: Harvard University Press.

Steyerl, Hito (Hg.) (2003) Spricht die Subalterne deutsch? Migration und postkoloniale Kritik. Münster: Unrast.

Schlehe, Judith (2002): Handeln und Aushandeln in transkulturellen Geschlechterbeziehungen. In: Hauser-Schäublin und Ulrich Braukämper (Hg.): Ethnologie der Globalisierung. Perspektiven kultureller Verflechtungen. S. 205-220. Berlin: Reimer. 